Journal of Applied AnALysis

Vol. 9, No. 2 (2003), pp. 211-223

\title{
ON NONCOERCIVE ELLIPTIC PROBLEMS WITH DISCONTINUITIES
}

\author{
N. HALIDIAS \\ Received September 16, 2002 and, in revised form, January 20, 2003
}

\begin{abstract}
In this paper using the critical point theory of Chang [4] for locally Lipschitz functionals we prove an existence theorem for noncoercive Neumann problems with discontinuous nonlinearities. We use the mountain-pass theorem to obtain a nontrivial solution.
\end{abstract}

\section{Introduction}

The problem under consideration is a Neumann elliptic boundary value problem with multivalued nonlinear boundary conditions. Let $Z \subseteq \mathbb{R}^{N}$ be a bounded domain with a $C^{1}$-boundary $\Gamma$

$$
\begin{cases}-\operatorname{div}\left(\|D x(z)\|^{p-2} D x(z)\right)=f(z, x(z)) & \text { a.e. on } Z \\ -\frac{\partial x}{\partial n_{p}} \in \partial j(z, \tau(x)(z)) & \text { a.e. on } \Gamma, 2 \leq p<\infty\end{cases}
$$

2000 Mathematics Subject Classification. 35J25, 35J60.

Key words and phrases. Discontinuous nonlinearities, critical point theory, locally Lipschitz functionals, mountain-pass theorem.

This work supported partially by a postdoctoral scholarship from the State Scholarship Foundation (I.K.Y.) of Greece.

ISSN 1425-6908（C) Heldermann Verlag. 
Here the boundary condition is in the sense of Kenmochi [12] and the operator $\tau$ is the trace operator in $W^{1, p}(Z)$. We do not assume here that the right-hand side is a Carathéodory function.

Many authors considered elliptic problems with non Carathéodory right hand side. For example Heikkila-Lakshmikantham [9] had used the method of upper and lower solution to obtain existence theorems for certain differential equations with discontinuous nonlinearities involving pseudomonotone operators but they need the existence of upper and lower solutions. On the other hand many authors established existence results for these problems without upper and lower solutions using the critical point theory for smooth or nonsmooth operators. Hence they need the differential operator to be of variational type. Some characteristic papers on this direction is that of Ambrosseti-Badiale [2] and Stuart-Tolland [15]. But non of them considered Neumann problems with multivalued boundary conditions or noncoercive problems. Here we consider noncoercive Neumann problems with multivalued boundary conditions and we do not use the method of upper and lower solution.

Also, for some existence results about the $p$-Laplacian with nonlinear boundary conditions see also [3].

For a more detailed study of the $p$-Laplacian one can read the well-written book of Drábek-Kufner-Nicolosi [8].

This problem is closely related with the so-called hemivariational inequalities. At this subject see [13], [14] and the references therein.

In the next section we recall some facts and definitions from the critical point theory for locally Lipschitz functionals and the subdifferential of Clarke.

\section{Preliminaries}

Let $X$ a real reflexive Banach space and let $Y$ be a subset of $X$. A function $f: Y \rightarrow \mathbb{R}$ is said to satisfy a Lipschitz condition (on $Y$ ) provided that, for some nonnegative scalar $K$, one has

$$
|f(y)-f(x)| \leq K\|y-x\|
$$

for all points $x, y \in Y$. Let $f$ be Lipschitz near a given point $x$, and let $v$ be any other vector in $X$. The generalized directional derivative of $f$ at $x$ in the direction $v$, denoted by $f^{0}(x ; v)$ is defined as follows:

$$
f^{0}(x ; v)=\limsup _{\substack{y \rightarrow x \\ t \downarrow 0}} \frac{f(y+t v)-f(y)}{t}
$$


where $y$ is a vector in $X$ and $t$ a positive scalar. If $f$ is Lipschitz of rank $K$ near $x$ then the function $v \rightarrow f^{0}(x ; v)$ is finite, positively homogeneous, subadditive and satisfies $\left|f^{0}(x ; v)\right| \leq K\|v\|$. In addition $f^{0}$ satisfies $f^{0}(x ;-v)=(-f)^{0}(x ; v)$. Now we are ready to introduce the generalized gradient which denoted by $\partial f(x)$ as follows:

$$
\partial f(x)=\left\{w \in X^{*}: f^{0}(x ; v) \geq\langle w, v\rangle \text { for all } v \in X\right\} .
$$

Some basic properties of the generalized gradient of locally Lipschitz functionals are the following:

(a) $\partial f(x)$ is a nonempty, convex, weakly compact subset of $X^{*}$ and $\|w\|_{*} \leq K$ for every $w$ in $\partial f(x)$.

(b) For every $v$ in $X$, one has

$$
f^{0}(x ; v)=\max \{\langle w, v\rangle: w \in \partial f(x)\} .
$$

If $f_{1}, f_{2}$ are locally Lipschitz functions then

$$
\partial\left(f_{1}+f_{2}\right) \subseteq \partial f_{1}+\partial f_{2} .
$$

Let us recall the (PS)-condition introduced by Chang.

Definition 1. We say that Lipschitz function $f$ satisfies the Palais-Smale condition if any sequence $\left\{x_{n}\right\} \subseteq X$ along which $\left|f\left(x_{n}\right)\right|$ is bounded and $\lambda\left(x_{n}\right)=\min _{w \in \partial f\left(x_{n}\right)}\|w\|_{X^{*}} \rightarrow 0$ possesses a convergent subsequence.

The (PS)-condition can also be formulated as follows (see Costa and Goncalves [6]).

(PS $)_{c,+}^{*}:$ Whenever $\left(x_{n}\right) \subseteq X,\left(\varepsilon_{n}\right),\left(\delta_{n}\right) \subseteq \mathbb{R}_{+}$are sequences with $\varepsilon_{n} \rightarrow 0, \delta_{n} \rightarrow 0$, and such that

$$
\begin{aligned}
& f\left(x_{n}\right) \rightarrow c \\
& f\left(x_{n}\right) \leq f(x)+\varepsilon_{n}\left\|x-x_{n}\right\| \text { if }\left\|x-x_{n}\right\| \leq \delta_{n},
\end{aligned}
$$

then $\left(x_{n}\right)$ possesses a convergent subsequence: $x_{n^{\prime}} \rightarrow \widehat{x}$.

Similarly, we define the $(\mathbf{P S})_{c}^{*}$ condition from below, $(\mathbf{P S})_{c,-}^{*}$, by interchanging $x$ and $x_{n}$ in the above inequality. And finally we say that $f$ satisfies $(\mathbf{P S})_{c}^{*}$ provided it satisfies $(\mathbf{P S})_{c,+}^{*}$ and $(\mathbf{P S})_{c,-}^{*}$.

Note that these two definitions are equivalent when $f$ is locally Lipschitz functional.

Theorem 1. If a locally Lipschitz functional $f: X \rightarrow \mathbb{R}$ on the reflexive Banach space $X$ satisfies the (PS)-condition and the hypotheses

(i) there exist positive constants $\rho$ and a such that

$$
f(u) \geq a \text { for all } u \in X \text { with }\|u\|=\rho \text {; }
$$


(ii) $f(0)=0$ and there a point $e \in X$ such that

$$
\|e\|>\rho \text { and } f(e) \leq 0
$$

then there exists a critical value $c \geq a$ of $f$ determined by

$$
c=\inf _{g \in G} \max _{t \in[0,1]} f(g(t))
$$

where

$$
G=\{g \in C([0,1], X): g(0)=0, g(1)=e\} .
$$

In what follows we will use the well-known inequality

$$
\sum_{j=1}^{N}\left(a_{j}(\eta)-a_{j}\left(\eta^{\prime}\right)\right)\left(\eta_{j}-\eta_{j}^{\prime}\right) \geq C\left|\eta-\eta^{\prime}\right|^{p}
$$

for $\eta, \eta^{\prime} \in \mathbb{R}^{N}$, with $a_{j}(\eta)=|\eta|^{p-2} \eta_{j}$.

\section{Neumann problems}

Let $X=W^{1, p}(Z)$. In the following we will need some definitions. Let $f: Z \times \mathbb{R} \rightarrow \mathbb{R}$. Then we introduce the following functions

$$
f_{1}(z, x)=\liminf _{x^{\prime} \rightarrow x} f\left(z, x^{\prime}\right), f_{2}(z, x)=\limsup _{x^{\prime} \rightarrow x} f\left(z, x^{\prime}\right) .
$$

Let $x$ satisfies the boundary conditions of problem (1).

Definition 2. We say that $x \in X$ is a solution of type I of problem (1) if there exists some $w \in L^{q}(Z)$ such that

$$
w(z) \in\left[f_{1}(z, x(z)), f_{2}(z, x(z))\right] \text { a.e. on } Z
$$

and

$$
-\operatorname{div}\left(\|D x(z)\|^{p-2} D x(z)\right)=w(z) \text { for almost all } z \in Z \text {. }
$$

Definition 3. We say that $x \in X$ is a solution of type II of problem (1) if

$$
-\operatorname{div}\left(\|D x(z)\|^{p-2} D x(z)\right)=f(z, x(z)) \text { for almost all } z \in Z \text {. }
$$

Our existence theorem concerns the existence of nontrivial solutions of type I. We use the mountain-pass theorem to obtain such a solution.

Our hypotheses on $f(z, x)$ and $j(z, x)$ are the following:

$\mathbf{H}(f): f_{1}, f_{2}: Z \times \mathbb{R} \rightarrow \mathbb{R}$ are $N$-measurable (i.e. if $x(z)$ is measurable then so are $\left.f_{1}(z, x(z)), f_{2}(z, x(z))\right)$;

(i) for almost all $z \in Z$ and all $x \in \mathbb{R},|f(z, x)| \leq \alpha(z)+c|x|^{\theta-1}$ with $\alpha \in L^{\infty}(Z), c>0,1 \leq \theta<p$ 
(ii) uniformly for almost all $z \in Z$ we have that

$$
\frac{f_{1,2}(z, x)}{|x|^{\theta-2} x} \rightarrow f_{+}(z) \text { as } x \rightarrow \pm \infty
$$

where $f_{+} \in L^{1}(Z), f_{+} \geq 0$ with strict inequality on a set of positive Lebesgue measure;

(iii) uniformly for almost all $z \in Z$ we have that

$$
\limsup _{x \rightarrow 0} \frac{p F(z, x)}{|x|^{p}} \leq h(z),
$$

with $h \in L^{\infty}(Z)$ and $h(z) \leq 0$ with strict inequality on a set of positive measure. Here, by $F(z, x)$ we denote the integral of $f$, that is $F(z, x)=$ $\int_{0}^{x} f(z, r) d r$.

$\mathbf{H}(j): j(z, x): Z \times \mathbb{R} \rightarrow \mathbb{R}$ is such that $z \rightarrow j(z, x)$ is measurable and $j(z, \cdot)$ is a locally Lipschitz function such that for almost all $z \in Z$, all $x \in \mathbb{R}$ and all $v(z) \in \partial j(z, x)$ we have that $|v| \leq \alpha_{1}(z)+c_{1}|x|^{\mu}, 0 \leq \mu<\theta-1$ ( $\theta$ the same as in $\mathbf{H}(f)(\mathrm{i}))$ with $\alpha_{1} \in L^{\infty}, c_{1}>0$ and $j(\cdot, 0) \in L^{\infty}(Z)$ and finally $j(z, \cdot) \geq 0$ for almost all $z \in Z$.

Theorem 2. If hypotheses $\mathbf{H}(f)$ and $\mathbf{H}(j)$ hold, then problem (1) has a nontrivial solution of type $I$.

Proof. Let $\Phi: W^{1, p}(Z) \rightarrow \mathbb{R}$ and $\psi: W^{1, p}(Z) \rightarrow \mathbb{R}_{+}$be defined by

$$
\Phi(x)=-\int_{Z} F(z, x(z)) d z \text { and } \psi(x)=\frac{1}{p}\|D x\|_{p}^{p}+\int_{\Gamma} j(z, \tau(x)(z)) d \sigma .
$$

In the definition of $\Phi(\cdot), F(z, x)=\int_{0}^{x} f(z, r) d r$ (the potential of $\left.f\right), \tau(\cdot)$ is the trace operator on $W^{1, p}(Z)$ and $d \sigma$ is the $(N-1)$-dimensional Hausdorff measure. Clearly $\Phi$, is locally Lipschitz (see Chang [4]), while we can check that $\psi$ is locally Lipschitz too. Set $R=\Phi+\psi$.

Claim 1. $R(\cdot)$ satisfies the (PS)-condition (in the sense of Costa and Goncalves). Let us first begin with (PS $)_{c,+}$.

Let $\left\{x_{n}\right\}_{n \geq 1} \subseteq W^{1, p}(Z)$ such that $R\left(x_{n}\right) \rightarrow c$ when $n \rightarrow \infty$ and

$$
R\left(x_{n}\right) \leq R(x)+\varepsilon_{n}\left\|x-x_{n}\right\| \text { with }\left\|x-x_{n}\right\| \leq \delta_{n} .
$$

with $\varepsilon_{n}, \delta_{n} \rightarrow 0$. This inequality is equivalent with

$$
R(x)-R\left(x_{n}\right) \geq-\varepsilon_{n}\left\|x-x_{n}\right\| \text { with }\left\|x-x_{n}\right\| \leq \delta_{n} .
$$

Choose $x=x_{n}+\delta x_{n}$ with $\delta\left\|x_{n}\right\| \leq \delta_{n}$. Divide with $\delta$. So, if $\delta \rightarrow 0$ we have that

$$
\lim _{\delta \rightarrow 0} \frac{R\left(x_{n}+\delta x_{n}\right)-R\left(x_{n}\right)}{\delta} \leq R^{0}\left(x_{n} ; x_{n}\right) .
$$

Thus we have that $R^{0}\left(x_{n} ; x_{n}\right) \geq-\varepsilon_{n}\left\|x_{n}\right\|$. 
For the $(\mathbf{P S})_{c,-}$ we have the following. Let $\left\{x_{n}\right\}_{n \geq 1} \subseteq W^{1, p}(Z)$ such that $R\left(x_{n}\right) \rightarrow c$ when $n \rightarrow \infty$ and

$$
R(x) \leq R\left(x_{n}\right)+\varepsilon_{n}\left\|x-x_{n}\right\| \text { with }\left\|x-x_{n}\right\| \leq \delta_{n} .
$$

with $\varepsilon_{n}, \delta_{n} \rightarrow 0$. This inequality is equivalent to the following

$$
(-R)(x)-(-R)\left(x_{n}\right) \geq-\varepsilon_{n}\left\|x-x_{n}\right\| \text { with }\left\|x-x_{n}\right\| \leq \delta_{n} .
$$

Note that $(-R)$ is locally Lipschitz too. Choose $x=x_{n}+\delta\left(-x_{n}\right)$ with $\delta\left\|x_{n}\right\| \leq \delta_{n}$. So if we divide with $\delta$ and take the limit when $\delta \rightarrow 0$ we have

$$
\lim _{\delta \rightarrow 0} \frac{(-R)\left(x_{n}+\delta\left(-x_{n}\right)\right)-(-R)\left(x_{n}\right)}{\delta} \leq(-R)^{0}\left(x_{n} ;\left(-x_{n}\right)\right)=R^{0}\left(x_{n} ; x_{n}\right) .
$$

So as before we have that $R^{0}\left(x_{n} ; x_{n}\right) \geq-\varepsilon_{n}\left\|x_{n}\right\|$.

Also,

$$
\frac{1}{p}\left\|D x_{n}+\delta D x_{n}\right\|_{p}^{p}-\frac{1}{p}\left\|D x_{n}\right\|_{p}^{p}=\frac{1}{p}\left\|D x_{n}\right\|_{p}^{p}\left((1+\delta)^{p}-1\right) .
$$

So if we divide this with $\delta$ and let $\delta \rightarrow 0$ we have that is equal with $\left\|D x_{n}\right\|_{p}^{p}$. Finally, there exists $w_{n} \in \partial \eta\left(x_{n}\right)$ where $\eta(x)=\int_{\Gamma} j(z, \tau(x)(z)) d \sigma$ such that $\eta^{0}\left(x_{n} ; x_{n}\right)=\int_{\Gamma} w_{n}(z) x_{n}(z) d \sigma$ and $v_{n}(z) \in\left[-f_{1}\left(z, x_{n}(z)\right),-f_{2}\left(z, x_{n}(z)\right)\right]$ such that $\left\langle v_{n}, x_{n}\right\rangle=\Phi^{0}\left(x_{n} ; x_{n}\right)$. Note that $w_{n}(z) \in \partial j\left(z, \tau\left(x_{n}\right)(z)\right)$ a.e. on $Z$. So, it follows that

$$
\int_{Z} v_{n} x_{n}(z) d z-\left\|D x_{n}\right\|_{p}^{p}-\int_{\Gamma} w_{n}(z) \tau\left(x_{n}\right)(z) d \sigma \leq \varepsilon_{n}\left\|x_{n}\right\|,
$$

for some $v_{n}(z) \in\left[f_{1}\left(z, x_{n}(z)\right), f_{2}\left(z, x_{n}(z)\right)\right]$.

If we choose for the $(\mathbf{P S})_{c,+}$, at the first place, $x=x_{n}+\delta\left(-x_{n}\right)$ we obtain $R^{0}\left(x_{n} ;-x_{n}\right) \geq-\varepsilon_{n}\left\|x_{n}\right\|$ and for the $(\mathbf{P S})_{c,-}$ we choose $x=x_{n}+\delta x_{n}$ to obtain the same inequality. But from $R^{0}\left(x_{n} ;-x_{n}\right) \geq-\varepsilon_{n}\left\|x_{n}\right\|$ we also obtain

$$
-\varepsilon_{n}\left\|x_{n}\right\| \leq \int_{Z} v_{n} x_{n}(z) d z-\left\|D x_{n}\right\|_{p}^{p}-\int_{\Gamma} w_{n}(z) \tau\left(x_{n}\right)(z) d \sigma \leq \varepsilon_{n}\left\|x_{n}\right\| .
$$

Suppose that $\left\{x_{n}\right\} \subseteq W^{1, p}(Z)$ was unbounded. Then (at least for a subsequence), we may assume that $\left\|x_{n}\right\| \rightarrow \infty$. Let $y_{n}=x_{n} /\left\|x_{n}\right\|, n \geq 1$. By passing to a subsequence if necessary, we may assume that

$$
\begin{array}{r}
y_{n} \stackrel{w}{\rightarrow} y \text { in } W^{1, p}(Z), y_{n} \rightarrow y \text { in } L^{p}(Z), y_{n}(z) \rightarrow y(z) \text { a.e. on } Z \text { as } n \rightarrow \infty \\
\text { and }\left|y_{n}(z)\right| \leq k(z) \text { a.e. on } Z \text { with } k \in L^{p}(Z) .
\end{array}
$$


Recall that from the choice of the sequence $\left\{x_{n}\right\}$ we have $\left|R\left(x_{n}\right)\right| \leq M_{1}$ for some $M_{1}>0$ and all $n \geq 1$,

$$
\begin{aligned}
& \Rightarrow \frac{1}{p}\left\|D x_{n}\right\|_{p}^{p}+\int_{\Gamma} j\left(z, \tau\left(x_{n}\right)(z)\right) d \sigma-\int_{Z} F\left(z, x_{n}(z)\right) d z \leq M_{1} \\
& \Rightarrow \frac{1}{p}\left\|D x_{n}\right\|_{p}^{p}-\int_{Z} F\left(z, x_{n}(z)\right) d z \leq M_{1}(\text { since } j \geq 0) .
\end{aligned}
$$

Divide by $\left\|x_{n}\right\|^{p}$. We obtain

$$
\frac{1}{p}\left\|D y_{n}\right\|_{p}^{p}-\int_{Z} \frac{F\left(z, x_{n}(z)\right)}{\left\|x_{n}\right\|^{p}} d z \leq \frac{M_{1}}{\left\|x_{n}\right\|^{p}} .
$$

We have

$$
\begin{aligned}
\left|\int_{Z} \frac{F\left(z, x_{n}(z)\right)}{\left\|x_{n}\right\|^{p}} d z\right| & \leq \frac{1}{\left\|x_{n}\right\|^{p}} \int_{Z} \int_{0}^{\left|x_{n}(z)\right|}|f(z, r)| d r d z \\
& \leq \frac{1}{\left\|x_{n}\right\|^{p}}\left(\|\alpha\|_{\infty}\left\|x_{n}\right\|+\frac{c}{\theta}\left\|x_{n}\right\|^{\theta}\right) \rightarrow 0 \text { as } n \rightarrow \infty .
\end{aligned}
$$

So by passing to the limit as $n \rightarrow \infty$ in (3), we obtain

$$
\begin{aligned}
\lim \frac{1}{p}\left\|D y_{n}\right\|_{p}^{p} & =0 \\
\Rightarrow\|D y\|_{p} & =0\left(\text { recall that } D y_{n} \stackrel{w}{\rightarrow} D y \text { in } L^{p}\left(Z, \mathbb{R}^{N}\right) \text { as } n \rightarrow \infty\right) \\
\Rightarrow y & =\xi \in \mathbb{R} .
\end{aligned}
$$

Note that $y_{n} \rightarrow \xi$ in $W^{1, p}(Z)$ and since $\left\|y_{n}\right\|=1, n \geq 1$ we infer that $\xi \neq 0$. We deduce that $\left|x_{n}(z)\right| \rightarrow+\infty$ a.e. on $Z$ as $n \rightarrow \infty$.

From the choice of the sequence $\left\{x_{n}\right\} \subseteq W^{1, p}(Z)$, we have

$$
\begin{aligned}
-\varepsilon_{n}\left\|x_{n}\right\| \leq & \int_{Z} v_{n}(z) x_{n}(z) d z-\left\|D x_{n}\right\|_{p}^{p} \\
& -\int_{Z} w_{n}(z) \tau\left(x_{n}\right)(z) d z \leq \varepsilon_{n}\left\|x_{n}\right\|
\end{aligned}
$$

and

$$
\begin{aligned}
-p M_{1} & \leq\left\|D x_{n}\right\|_{p}^{p}+p \int_{\Gamma} j\left(z, \tau(x)(z) d \sigma-p \int_{Z} F\left(z, x_{n}(z)\right) d z\right. \\
& \leq p M_{1} .
\end{aligned}
$$

Substituting (4) and (5), we obtain

$$
\begin{aligned}
& -p M_{1}-\varepsilon_{n}\left\|x_{n}\right\| \leq \int_{\Gamma}\left(p j\left(z, \tau\left(x_{n}\right)(z)\right)-w_{n}(z) \tau\left(x_{n}\right)(z) d \sigma\right. \\
& \left.+\int_{Z}\left(v_{n}(z)\right) x_{n}(z)-p F\left(z, x_{n}(z)\right)\right) d z \leq p M_{1}+\varepsilon_{n}\left\|x_{n}\right\| .
\end{aligned}
$$


Divide this inequality by $\left\|x_{n}\right\|^{\theta}$. We have

$$
\begin{aligned}
& \int_{Z} \frac{v_{n}(z)}{\left\|x_{n}\right\|^{\theta-1}} y_{n}(z) d z-\int_{Z} \frac{p F\left(z, x_{n}(z)\right)}{\left\|x_{n}\right\|^{\theta}} d z \\
& +\int_{\Gamma} \frac{p j\left(z, \tau\left(x_{n}\right)(z)-w_{n}(z) \tau\left(x_{n}\right)(z)\right.}{\left\|x_{n}\right\|^{\theta}} d \sigma \\
& \geq \frac{1}{\left\|x_{n}\right\|^{\theta}} p M_{1}+\frac{\varepsilon_{n}}{\left\|x_{n}\right\|^{\theta-1}} .
\end{aligned}
$$

Note that

$$
\int_{Z} \frac{v_{n}(z)}{\left\|x_{n}\right\|^{\theta-1}} y_{n}(z) d z=\int_{Z} \frac{v_{n}(z)}{\left|x_{n}(z)\right|^{\theta-2} x_{n}(z)}\left|y_{n}(z)\right|^{\theta} d z \rightarrow|\xi|^{\theta} \int_{Z} f_{+}(z) d z
$$

as $n \rightarrow \infty$.

Also by virtue of hypothesis $\mathbf{H}(f)$ (ii), given $z \in Z \backslash N,|N|=0(|C|$ denotes the Lebesgue measure of a measurable set $C \subseteq Z$ ) and $\varepsilon>0$, we can find $M_{\varepsilon}>0$ such that for all $|r| \geq M_{\varepsilon}$ we have

$$
\left|f_{+}(z)-\frac{f_{1,2}(z, r)}{|r|^{\theta-2} r}\right| \leq \varepsilon
$$

Then, if $x_{n}(z) \rightarrow+\infty$, we have

$$
\begin{aligned}
\frac{1}{\left|x_{n}(z)\right|^{\theta}} F\left(z, x_{n}(z)\right) \geq & \frac{1}{\left|x_{n}(z)\right|^{\theta}} F\left(z, M_{\varepsilon}\right) \\
& +\frac{1}{\left|x_{n}(z)\right|^{\theta}} \int_{M_{\varepsilon}}^{x_{n}(z)}\left(f_{+}(z)|r|^{\theta-2} r-\varepsilon|r|^{\theta-2} r\right) d r \\
= & \frac{1}{\left|x_{n}(z)\right|^{\theta}} \eta(z)+\frac{\left|x_{n}(z)\right|^{\theta}-M_{\varepsilon}^{\theta}}{\theta\left|x_{n}(z)\right|^{\theta}}\left(f_{+}(z)-\varepsilon\right) \\
& \text { for some } \eta \in L^{1}(Z) \\
\Rightarrow \liminf _{n \rightarrow \infty} \frac{F\left(z, x_{n}(z)\right)}{\left|x_{n}(z)\right|^{\theta}} \geq & \frac{1}{\theta}\left(f_{+}(z)-\varepsilon\right) .
\end{aligned}
$$

Similarly we obtain that

$$
\limsup _{n \rightarrow \infty} \frac{F\left(z, x_{n}(z)\right)}{\left|x_{n}(z)\right|^{\theta}} \leq \frac{1}{\theta}\left(f_{+}(z)+\varepsilon\right)
$$


From (7) and (8) and since $\varepsilon>0$ and $z \in Z \backslash N$ were arbitrary, we infer that

$$
\begin{aligned}
& \frac{F\left(z, x_{n}(z)\right)}{\left|x_{n}(z)\right|^{\theta}} \rightarrow \frac{1}{\theta} f_{+}(z) \text { a.e. on } Z \text { as } n \rightarrow \infty \\
& \Rightarrow \int_{Z} \frac{F\left(z, x_{n}(z)\right)}{\| x_{n}||^{\theta}} d z=\int_{Z} \frac{F\left(z, x_{n}(z)\right)}{\left|x_{n}(z)\right|^{\theta}} \frac{\left|x_{n}(z)\right|^{\theta}}{\| x_{n}||^{\theta}} d z \\
& =\int_{Z} \frac{F\left(z, x_{n}(z)\right)}{\left|x_{n}(z)\right|^{\theta}}\left|y_{n}(z)\right|^{\theta} d z \rightarrow \xi^{\theta} \int_{Z} \frac{1}{\theta} f_{+}(z) \text { as } n \rightarrow \infty .
\end{aligned}
$$

Note that for almost all $z \in Z j(z, \cdot)$ is locally Lipschitz. So by Lebourg's mean value theorem (see Clarke [5, Theorem 2.3.7, p. 41]), for almost all $z \in Z$ and all $x \in \mathbb{R}$, we can find $w(z) \in \partial j(z, \eta x) 0<\eta<1$ such that

$$
\begin{gathered}
|j(z, x)-j(z, 0)|=w(z) x \\
\Rightarrow|j(z, x)| \leq|j(z, 0)|+|w(z)||x| \leq \beta+|w(z)||x|\left(\text { since } j(\cdot, 0) \in L^{\infty}(Z)\right) .
\end{gathered}
$$

But by $\mathbf{H}(j)$ we have

$$
\begin{aligned}
|w(z)| & \leq a_{1}(z)+c_{1}|x|^{\mu} \\
\Rightarrow|j(z, x)| & \leq c_{1}+c_{2}|x|+c_{3}|x|^{\mu+1} \text { for some } c_{1}, c_{2}, c_{3}>0 .
\end{aligned}
$$

So it is easy to see that

$$
\int_{\Gamma} \frac{p j\left(z, \tau\left(x_{n}\right)(z)\right)-w_{n}(z) \tau\left(x_{n}\right)(z)}{\left\|x_{n}\right\|^{\theta}} d \sigma \rightarrow 0 \text { as } n \rightarrow \infty
$$

(recall that $\mu+1<\theta$ ).

Indeed,

$$
\begin{aligned}
& \int_{\Gamma} \frac{p j\left(z, \tau\left(x_{n}\right)(z)\right)-w_{n}(z) \tau\left(x_{n}\right)(z)}{\left\|x_{n}\right\|^{\theta}} d \sigma \\
& \leq \int_{\Gamma} \frac{c_{2}\left|x_{n}(z)\right|}{\left\|x_{n}\right\|^{\theta}} d \sigma+\int_{\Gamma} \frac{c_{3}\left|x_{n}(z)\right|^{\mu+1}}{\left\|x_{n}\right\|^{\theta}}+\frac{c_{1}}{\left\|x_{n}\right\|^{\theta}} \\
& \leq c_{2} \frac{\left\|x_{n}\right\|_{L^{1}(\Gamma)}}{\left\|x_{n}\right\|^{\theta}}+c_{3} \frac{\left\|x_{n}\right\|_{L^{\mu+1}(\Gamma)}^{\mu+1}}{\left\|x_{n}\right\|^{\theta}}+\frac{c_{1}}{\left\|x_{n}\right\|^{\theta}} .
\end{aligned}
$$

But we know that

$$
\begin{aligned}
& \left\|x_{n}\right\|_{L^{1}(\Gamma)} \leq C\left\|x_{n}\right\|_{\frac{1}{q}, 1, \Gamma} \leq C\left\|x_{n}\right\|_{1, p, Z} \quad \text { and } \\
& \left\|x_{n}\right\|_{L^{\mu+1}(\Gamma)}^{\mu+1} \leq C\left\|x_{n}\right\|_{\frac{1}{q}, p, \Gamma}^{\mu+1} \leq C\left\|x_{n}\right\|_{1, p, Z}^{\mu+1}, \quad \text { see Adams [1, p. 217]). }
\end{aligned}
$$

Thus by passing to the limit in (6), we obtain

$$
\left(1-\frac{p}{\theta}\right) \xi^{\theta} \int_{Z} f_{+}(z) \geq 0
$$


a contradiction to hypothesis $\mathbf{H}(f)$ (ii) (recall $p>\theta$ ). If $x_{n}(z) \rightarrow-\infty$, with similar arguments as above we show that

$$
\int_{Z} \frac{F\left(z, x_{n}(z)\right)}{\left\|x_{n}\right\|^{\theta}} d z \rightarrow \xi^{\theta} \int_{Z} \frac{1}{\theta} f_{+}(z) \text { as } n \rightarrow \infty .
$$

Therefore it follows that $\left\{x_{n}\right\} \subseteq W^{1, p}(Z)$ is bounded. Hence we may assume that $x_{n} \stackrel{w}{\rightarrow} x$ in $W^{1, p}(Z), x_{n} \rightarrow x$ in $L^{p}(Z), x_{n}(z) \rightarrow x(z)$ a.e. on $Z$ as $n \rightarrow \infty$ and $\left|x_{n}(z)\right| \leq k(z)$ a.e. on $Z$ with $k \in L^{p}(Z)$.

From the properties of the subdifferential of Clarke, we have

$$
\begin{aligned}
& \partial R\left(x_{n}\right) \subseteq \partial \Phi\left(x_{n}\right)+\partial \psi\left(x_{n}\right) \\
& \subseteq \partial \Phi\left(x_{n}\right)+\partial\left(\frac{1}{p}\left\|D x_{n}\right\|_{p}^{p}\right)+\int_{\Gamma} \partial j\left(z, \tau\left(x_{n}(z)\right) d \sigma\right. \text { (see Clarke [5, p. 83]). }
\end{aligned}
$$

So we have

$$
\left\langle w_{n}, y\right\rangle=\left\langle A x_{n}, y\right\rangle+\left\langle r_{n}, y\right\rangle_{\Gamma}-\int_{Z} v_{n}(z) y(z) d z
$$

with $r_{n}(z) \in \partial j\left(z, \tau\left(x_{n}\right)(z)\right), v_{n}(z) \in\left[f_{1}\left(z, x_{n}(z)\right), f_{2}\left(z, x_{n}(z)\right)\right]$ and $w_{n}$ the element with minimal norm of the subdifferential of $R$ and $A: W^{1, p}(Z) \rightarrow$ $W^{1, p}(Z)^{*}$ such that $\langle A x, y\rangle=\int_{Z}\left(\|D x(z)\|^{p-2}(D x(z), D y(z))_{R^{N}} d z\right.$. But $x_{n} \stackrel{w}{\rightarrow} x$ in $W^{1, p}(Z)$, so $x_{n} \rightarrow x$ in $L^{p}(Z)$ and $x_{n}(z) \rightarrow x(z)$ a.e. on $Z$ by virtue of the compact embedding $W^{1, p}(Z) \subseteq L^{p}(Z)$. Note that the trace of $x_{n}$ belongs to $W^{1 / q, p}(\Gamma)$, so from $\mathbf{H}(j)$ we have that the trace of $r_{n}$ belongs in $L^{q}(\Gamma)$. But we have that $\tau\left(x_{n}\right)$ is bounded in $W^{1 / q, p}(\Gamma)$. Thus, $r_{n}$ is bounded in $L^{q}(\Gamma)$. Choose $y=x_{n}-x$. Due to the compactness of the trace operator $\tau: W^{1, p}(Z) \rightarrow L^{p}(\Gamma)$ we have that

$$
\left|\left\langle r_{n}, \tau\left(x_{n}-x\right)\right\rangle_{\Gamma}\right|=\left|\left(r_{n}, \tau\left(x_{n}-x\right)\right)_{\Gamma}\right| \rightarrow 0 .
$$

With $\langle\cdot, \cdot\rangle_{\Gamma}$ we denote the natural pairing of $\left(W^{1 / q, p}(\Gamma),\left(W^{1 / q, p}(\Gamma)\right)^{*}\right)$ and by $(\cdot, \cdot)_{\Gamma}$ the natural pairing of $\left(L^{p}(\Gamma), L^{q}(\Gamma)\right)$.

Then in the limit we have that $\lim \sup \left\langle A x_{n}, x_{n}-x\right\rangle=0$ (note that $v_{n}$ is bounded). By virtue of the inequality (2) we have that $D x_{n} \rightarrow D x$ in $L^{p}(Z)$. So we have $x_{n} \rightarrow x$ in $W^{1, p}(Z)$. The Claim is proved.

Now for every $\xi \in \mathbb{R}$ we have

$$
\begin{aligned}
R(\xi) & =\int_{\Gamma} j(z, \xi) d \sigma-\int_{Z} F(z, \xi) d z \\
& \leq\left\|a_{1}\right\|_{\infty}|\xi||\Gamma|+\frac{c_{1}}{\mu}|\xi|^{\mu}|\Gamma|-\int_{Z} F(z, \xi) d z \\
\Rightarrow \frac{1}{|\xi|^{\mu}} R(\xi) & \leq \frac{1}{|\xi|^{\mu-1}}\left\|a_{1}\right\|_{\infty}|\Gamma|+\frac{c}{\mu}|\Gamma|-\frac{1}{|\xi|^{\mu}} \int_{Z} F(z, \xi) d z .
\end{aligned}
$$

From hypotheses $\mathbf{H}(f)$ (ii) we conclude that $R(\xi) \rightarrow-\infty$ as $|\xi| \rightarrow \infty$. 
In order to use the mountain-pass theorem it remains to show that there exists $\rho>0$ such that for $\|x\|=\rho$ we have that $R(x) \geq a>0$. In fact, we will show that for every sequence $\left\{x_{n}\right\} \subseteq W^{1, p}(Z)$ with $\left\|x_{n}\right\|=\rho_{n} \downarrow 0$ we have that $R\left(x_{n}\right)>0$. Indeed, suppose not. Then there exists some sequence $\left\{x_{n}\right\}$ such that $R\left(x_{n}\right) \leq 0$. Thus, we have

$$
\frac{1}{p}\left\|D x_{n}\right\|_{p}^{p} \leq \int_{Z} F\left(z, x_{n}(z)\right) d z
$$

recall that $j \geq 0$.

Divide this inequality with $\left\|x_{n}\right\|^{p}$. Let $y_{n}(z)=x_{n}(z) /\left\|x_{n}\right\|$. Then we have

$$
\left\|D y_{n}\right\|_{p}^{p} \leq \int_{Z} p \frac{F\left(z, x_{n}(z)\right)}{\left\|x_{n}\right\|^{p}} d z .
$$

From $\mathbf{H}(f)$ (iii) we have that for almost all $z \in Z$ for any $\varepsilon>0$ we can find $\delta>0$ such that for $|x| \leq \delta$ we have

$$
p F(z, x) \leq(h(z)+\varepsilon)|x|^{p} .
$$

On the other hand, for almost all $z \in Z$ and all $|x| \geq \delta$ we have

$$
p|F(z, x)| \leq c_{1}|x|+c_{2}|x|^{\theta}+c_{3} \leq c_{1}|x|^{p}+c_{2}|x|^{p^{*}}+c_{4} .
$$

Thus we can always find $\gamma>0$ such that $p|F(z, x)| \leq(h(z)+\varepsilon)|x|^{p}+\gamma|x|^{p^{*}}$ for all $x \in \mathbb{R}$. Indeed, choose

$$
\gamma \geq c_{2}+\frac{c_{4}}{|\delta|^{p^{*}}}+\left|h(z)+\varepsilon-c_{1}\right||\delta|^{p-p^{*}} .
$$

Therefore, we obtain

$$
\begin{aligned}
\left\|D y_{n}\right\|_{p}^{p} & \leq \int_{Z}(h(z)+\varepsilon)\left|y_{n}(z)\right|^{p} d z+\gamma \int_{Z} \frac{\left|x_{n}(z)\right|^{p^{*}}}{\left\|x_{n}\right\|^{p}} d z \\
& \leq \int_{Z}(h(z)+\varepsilon)\left|y_{n}(z)\right|^{p} d z+\gamma_{1}\left\|x_{n}\right\|^{p^{*}-p} .
\end{aligned}
$$

Here we have used the fact that $W^{1, p}(Z)$ embeds continuously in $L^{p^{*}}(Z)$.

So we obtain

$$
0 \leq\left\|D y_{n}\right\|_{p}^{p} \leq \varepsilon\left\|y_{n}\right\|_{p}^{p}+\gamma_{1}\left\|x_{n}\right\|^{p^{*}-p} \text { recall that } h(z) \leq 0 .
$$

Therefore in the limit we have that $\left\|D y_{n}\right\|_{p} \rightarrow 0$. Recall that $y_{n} \rightarrow y$ weakly in $W^{1, p}(Z)$. So $\|D y\|_{p} \leq \liminf \left\|D y_{n}\right\|_{p} \leq \lim \sup \left\|D y_{n}\right\|_{p} \rightarrow 0$. So $\|D y\|_{p}=0$, thus $y=\xi \in \mathbb{R}$. Note that $D y_{n} \rightarrow D y$ weakly in $\left(L^{p}(Z)\right)^{N}$ and $\left\|D y_{n}\right\|_{p} \rightarrow\|D y\|_{p}$ so $y_{n} \rightarrow y$ in $W^{1, p}(Z)$. Since $\left\|y_{n}\right\|=1$ we have that $\|y\|=1$ so $\xi \neq 0$. Suppose that $\xi>0$. Going back to (10) we have

$$
0 \leq \int_{Z}(h(z)+\varepsilon) y_{n}^{p}(z) d z+\gamma_{1}\left\|x_{n}\right\|^{p^{*}-p} .
$$


In the limit we have

$$
0 \leq \int_{Z}(h(z)+\varepsilon) \xi^{p} d z \leq \varepsilon \xi^{p}|Z| \text { recall that } h(z) \leq 0 .
$$

Thus we obtain that $\int_{Z} h(z) \xi^{p} d z=0$. But this is a contradiction. The same holds when $\xi<0$. So the claim is proved.

Hence by Theorem 1 we have that there exists $x \in W^{1, p}(Z)$ such that $0 \in \partial R(x)$. That is $0 \in \partial \Phi(x)+\partial \psi(x)$.

So, we can say that

$$
\begin{aligned}
\int_{Z} w(z) y(z)= & \int_{Z}\|D x(z)\|^{p-2}(D x(z), D y(z)) d z \\
& +\int_{\Gamma} v(z) y(z) d \sigma
\end{aligned}
$$

for some $w \in L^{q}(Z)$ such that $w(z) \in\left[f_{1}(z, x(z)), f_{2}(z, x(z))\right]$ (note that $\partial(-\Phi)(x) \subseteq\left[f_{1}(z, x(z)), f_{2}(z, x(z))\right]$, see Chang [4]) and $v(z) \in \partial j(z, x(z))$ for every $y \in W^{1, p}(Z)$. Choose now $y=s \in C_{0}^{\infty}(Z)$. We obtain

$$
\int_{Z} w(z) s(z)=\int_{Z}\|D x(z)\|^{p-2}(D x(z), D s(z)) d z .
$$

Then we have that $\operatorname{div}\left(\|D x(z)\|^{p-2} D x(z)\right) \in L^{q}(Z)$ because $w \in L^{q}(Z)$.

Going back to (11) and letting $y=C^{\infty}(Z)$ and finally using the Green formula 1.6 of Kenmochi [12], we have that

$$
-\frac{\partial x}{\partial n_{p}} \in \partial j(z, \tau(x)(z)) \text {. }
$$

So $x$ is of type $\mathrm{I}$.

Remark 1. The question whenever there exists a solution of type II remains open.

\section{References}

[1] Adams, R. A., Sobolev Spaces, Academic Press, New York, 1975.

[2] Ambrosetti, A., Badiale, M., The dual variational principle and elliptic problems with discontinuous nonlinearities, J. Math. Anal. Appl. 140(2) (1989), 363-273.

[3] Bonder, J. F., Rossi, J. D., Existence results for the p-Laplacian with nonlinear boundary conditions, J. Math. Anal. Appl. 263 (2001), 195-223.

[4] Chang, K. C., Variational methods for non-differentiable functionals and their applications to partial differential equations, J. Math. Anal. Appl. 80 (1981), 102-129.

[5] Clarke, F., Optimization and Nonsmooth Analysis, John Wiley \& Sons, Inc., New York, 1983.

[6] Costa, D. G., Goncalves, J. V., Critical point theory for nondifferentiable functionals and applications, J. Math. Anal. Appl. 153 (1990), 470-485. 
[7] de Figueiredo, D. G., Lectures on the Ekeland Variational Principle with Applications and Detours, Lectures on Mathematics and Physics 81, Tata Institute of Fundamental Research, Bombay, Sringer-Verlag, Berlin, 1989.

[8] Drábek, P., Kufner, A., Nicolosi, F., Quasilinear Elliptic Equations with Degenerations and Singularities, de Gruyter Series in Nonlinear Analysis and Applications 5, Walter de Gruyter \& Co., Berlin, 1997.

[9] Heikkila, S., Lakshikantham, V., Monotone Iterative Techniques for Discontinuous Nonlinear Differential Equations, Marcel Dekker, 1994.

[10] Hu, S., Papageorgiou, N. S., Handbook of Multivalued Analysis. Volume I: Theory, Kluwer Academic Publishers, Dordrecht, 1997.

[11] Hu, S., Papageorgiou, N. S., Handbook of Multivalued Analysis. Volume II: Applications, Kluwer Academic Publishers, Dordrecht, 2000.

[12] Kenmochi, N., Pseudomonotone operators and nonlinear elliptic boundary value problems, J. Math. Soc. Japan 27(1) (1975), 121-149.

[13] Motreanu, D., Naniewicz, Z., Discontinuous semilinear problems in vector-valued function spaces, Differential Integral Equations 9 (1996), 581-598.

[14] Motreanu, D., Naniewicz, Z., A topological approach to hemivariational inequalities with unilateral growth condition, J. Appl. Anal. 7 (2001), 23-41.

[15] Stuart, C. A., Tolland, J. F., A variational method for boundary value problems with discontinuous nonlinearities, J. London Math. Soc. (2) 21 (1980), 319-328.

\author{
Nikolaos Halidias \\ UNIVERSity OF THE AEgEAN \\ Department of Statistics and Actuarial Science \\ KARLOVASSI, 83200 \\ Samos, Greece \\ NICK@AEGEAN.GR
}

